\title{
AUTOMATIC GENERATION OF NOISE-FREE TIME-ACTIVITY CURVE WITH GATED BLOOD-POOL EMISSION TOMOGRAPHY USING DEFORMATION OF A REFERENCE CURVE
}

\author{
Charles Caderas de Kerleau, Jean-Francois Crouzet, Ehoud Ahronovitz, \\ Michel Rossi, Denis MARIANO-GoularT
}

\begin{abstract}
This paper describes a new method for assessing clinical parameters from a noisy regional time-activity curve (TAC) in tomographic gated blood-pool ventriculography. This method is based on a priori knowledge on the shape of a TAC, and shape approximation. The rejection method was used to generate different random Poisson deviates, covering standard count levels, of six representative TACs in order to test and compare the proposed method with harmonic and multi-harmonic reconstruction methods. These methods were compared by evaluating four clinical parameters: time of end systole, amplitude, peak ejection and filling rates. Overall, the accuracy of assessment of these parameters was found to be better with the method described in this paper than with standard multi-harmonic fits.

Index Terms- Gated blood-pool SPECT, Wall motion analysis, Time activity curve, Deformable model fit.
\end{abstract}

\section{Introduction}

Tomographic gated blood-pool ventriculography (TRVG) is a technically simple and widely available count-based method used for the assessment of cardiac function. This method is based on measurement of the change in the amount of cardiac blood-pool activity with time, due to the change in the size of a given region of interest or to partial volume effects. These count-based methods were shown to be more accurate than direct methods which are designed to localize the ventricular wall and measure lengths or volumes [1]. TRVG permits simultaneous measurement at equilibrium of right and left ventricular ejection fractions, volumes and outputs, regurgitant fractions, and regional wall motion in addition to phase analysis $[2,3,4,5,6,7,8,9,10,11,12,13,14,15,16,17]$. Recently, software dedicated to the segmentation of TRVG data has been developed and validated, thus enhancing the potencial for widespread use of this technique in the clinical setting [18, 19, 20, 21, 22, 23, 24, 25, 26, 27, 28] - An optimal three-dimensional method for the generation of right and left ventricular time-activity curves (TAC) was described in a recent study [29]. The "radial method" proposed by Vilain et al [29] 
can be used to generate TACs for each surface voxel, with its activity being calculated as the sum of the activity of all cubic voxels of a radius traced between the corresponding ventricular surface and a defined ventricular center. This enables a more refined analysis of ventricular TACs for each voxel or group of voxels on the surface of a segmented ventricle, including evaluation of local ejection fractions (EF) and time of end systole (TES) as well as peak ejection (PER) and filling rates (PFR). However, a TAC representing the behavior of a single voxel on the surface of a ventricle is generated from a limited number of counts and substantial errors due to noise can be present.

When analyzing the complete left ventricle using isotopic planar equilibrium ventriculography, ejection and filling parameters are best obtained by performing a polynomial or a multi-harmonic Fourier fit on the TAC [30, 31, 32]. Multi-harmonic Fourier analysis has also been performed on pixel TACs in a region of interest containing the left ventricle, and good mathematical fits of the radionuclide data were obtained [33]. However, when an accurate 3-dimensional analysis of ventricular wall motion is necessary, multi-harmonic filtering may be unsuitable because of the noise level of the TAC derived for each voxel on the surface of the ventricles.

The aim of this study was to develop a new method for assessing cardiac function from TAC acquired using gated blood-pool emission tomography. This new method is based on the use of a priori knowledge on the shape of a time activity curve. This method was compared to standard clinical harmonic filtering methods using the accuracy of parameters derived from the fitted TAC.

\section{Theory}

Here we propose to derive the ejection fraction (EF), time of end systole (TES) and peak ejection and filling rate (PER and PFR) after fitting a noisy TAC, $E(t)$ by suitable deformation of a reference TAC. Simulated time-activity curves were scaled and reordered so that the temporal duration of the heart cycle was fixed at $1 \mathrm{sec}$ and the maximum activity on the curve was fixed at 1 count per region of interest and obtained for the first time sample. This is not a restrictive procedure as any experimental clinical TAC can be scaled and reordered in this way using suitable scaling factors for activities, times and for the clinical measurements derived from the TAC.

\subsection{Model of the reference TAC}

A reference TAC model was designed to represent the systolic and diastolic function of a normal ventricle. Five points were selected on a normal TAC. Three points corresponded to: 1) the beginning of ventricular systole, 2) the end of ventricular systole, and 3) the end of auricular systole (audible sound when valves between ventricles and atria open). The other two points were located in the last third of the TAC corresponding to diastasis and arterial contraction. A continuous periodic model $P(t)$ was created using periodic cubic spline interpolation of these samples. This model presents the following characteristics on its period (Fig. 1):

$$
P(t):[0,1] \rightarrow[0,1]
$$




$$
\begin{gathered}
P(0)=P(1)=1 \\
\frac{d P}{d t}(0)=\frac{d P}{d t}(1)=0 \\
\left.\exists ! t_{\text {min }} \in\right] 0,1\left[/ \frac{d P}{d t}\left(t_{\text {min }}\right)=0\right.
\end{gathered}
$$

and $\forall t \in] 0,1[$,

$$
\begin{aligned}
& t<t_{\text {min }} \Rightarrow \frac{d P}{d t}(t)<0 \\
& t>t_{\text {min }} \Rightarrow \frac{d P}{d t}(t)>0
\end{aligned}
$$

\subsection{Reshaping the model}

We will use two ways to reshape the model $P(t)$ without losing the properties defined by (1). First, as $P\left(t_{\min }\right)=1-\mathrm{EF}$, any EF can be associated to the model by using the following transformation:

$$
D(t)=P(t)^{\beta} \text { with } \beta=\frac{\log (1-\mathrm{EF})}{\log \left(P\left(t_{\min }\right)\right)}
$$

Where $D(t)$ represents the deformed curve. Given this transformation, we are able to force $D(t)$ to have any TES, PER and PFR. This can be done by replacing the variable $t$ by a bijective continuous time polynomial function, $Q(t)$. This polynomial function is meant to reshape the model $D(t)$. Thus, we redefine the modified model $D(t)$ as follows:

$$
\begin{aligned}
& D(t)=P[Q(t)]^{\beta} \\
& Q(t):[0,1] \rightarrow[0,1], \\
& \text { with } Q(t) \text { being an increasing polynomial function such that } \\
& Q(0)=0 \text { and } Q(1)=1 .
\end{aligned}
$$

\subsection{Modeling an experimental TAC}

Having described the development of the model $P(t)$ and a way to reshape it, we turn our attention to the use of this model. Our purpose is to smooth and interpolate a noisy TAC defined by T samples, $E\left(t_{i}\right), i=1,2, \ldots \mathrm{T}$, where $\mathrm{T}$ is the number of intervals acquired per cardiac cycle.

\subsubsection{First estimation of EF and TES}

A rough estimation of the time of end systole, TES est $_{\text {of }} E\left(t_{i}\right)$ is first calculated as follows: a periodic spline interpolation of $E\left(t_{i}\right)$ is computed to provide an estimate of the ejection fraction $\mathrm{EF}_{\text {est }}$. Substituting $\mathrm{EF}_{\text {est }}$ in (2) provides a first $\left(\beta_{\text {est }}\right)$ for $\beta$. We then search for the two lowest values of $E\left(t_{i}\right)$, and 
if they occur consecutively TES $_{\text {est }}$ is set at the absolute minimum of the previously computed spline interpolation. Otherwise, measured data points in the neighborhood of the TAC minimum are too noisy to provide an accurate estimation of the TES using a simple spline interpolation of $\left(t_{i}, E\left(t_{i}\right)\right)$. Thus, the intercorrelation function $I(k)=\sum_{i=1}^{T} E\left(t_{i}\right) P\left(t_{i+k}\right)^{\beta_{\text {est }}}$ is computed and TES $\mathrm{S}_{\text {est }}$ is set at the time of end systole of the function $P\left(t_{i+j}\right)^{\beta_{\text {est }}}$, where $I(j)=\operatorname{MAX} X_{k \in[0, T[} I(k)$.

\subsubsection{Key points}

By definition $D(t)$ is built with the help of a model $P(t)$, a $\beta$ value which controls EF and a polynomial $Q(t)$ which controls the global modified shape. If the experimental data were noise free, the polynomial values $Q\left(t_{i}\right)=t_{i}^{\prime}$ for each acquired time sample $t_{i}$ could be computed as follows (Fig. 2):

$\forall i \in[1, T], \exists !\left(u_{i}, v_{i}\right) \in[0,1]^{2}$ such that

$E\left(t_{i}\right)=P\left(u_{i}\right)^{\beta}=P\left(v_{i}\right)^{\beta}$ and $u_{i} \leq v_{i}$

if $t_{i} \leq T E S_{\text {est }}$ then $t_{i}^{\prime}=u_{i}$

if $t_{i} \geq T E S_{\text {est }}$ then $t_{i}^{\prime}=v_{i}$

Each point $\left(t_{i}, t_{i}^{\prime}\right)$ will be called a "key point", and the set of all key points will be $\mathcal{K}=\left\{\left(t_{i}, t_{i}^{\prime}\right), i=\right.$ $1,2, \ldots, T\}$

\subsubsection{Improving the $\beta$ value}

We are now going to prove that the $\beta$ value and key points can lead to a better estimation of $\beta$. We are now going to prove that the $\beta$ value and key points derived from experimental noisy data are linked so that a more accurate estimation of key points can lead to a better estimation of $\beta$.

Key points and $\beta$ value: Let us have a closer look at how the behavior of key points depends on the value of $\beta$. If $\left(t_{i}, E\left(t_{i}\right)\right)$ is an acquired point, its associated key point $\left(t_{i}, t_{i}^{\prime}\right)$ is such that $P\left(t_{i}^{\prime}\right)^{\beta}=E\left(t_{i}\right)$. For another value of $\beta\left(\beta^{\prime}\right)$, the associated key point $\left(t_{i}, t_{i}^{\prime \prime}\right)$ is such that $P\left(t_{i}^{\prime \prime}\right)^{\beta^{\prime}}=E\left(t_{i}\right)$.

So, we have $P\left(t_{i}^{\prime}\right)^{\beta}=E\left(t_{i}\right)=P\left(t_{i}^{\prime \prime}\right)^{\beta^{\prime}}$.

Let $\beta^{\prime}>\beta$, then

$P\left(t_{i}^{\prime}\right)^{\beta}=P\left(t_{i}^{\prime \prime}\right)^{\beta^{\prime}}<P\left(t_{i}^{\prime \prime}\right)^{\beta} \Rightarrow P\left(t_{i}^{\prime}\right)<P\left(t_{i}^{\prime \prime}\right)$

if $t_{i}<T E S_{\text {est }}$, then $t_{i}^{\prime \prime}<t_{i}^{\prime}$, because the polynomial function $P$ is decreasing in the neighborhood of $t_{i}$;

if $t_{i}>T E S_{\text {est }}$, then $t_{i}^{\prime \prime}>t_{i}^{\prime}$, because the polynomial function $P$ is increasing in the neighborhood of $t_{i}$;

and conversely if $\beta^{\prime}<\beta$. Consequently, adjusting the $\beta$ value is a way to increase or decrease the ordinates of key points, depending on their position with respect to TES ${ }_{\text {est }}$

Smoothness: The deformed model $D(t)$ is expected to have the same general shape as the reference TAC $P(t)$. A suitable approximation of the key points $\left(t_{i}, t_{i}^{\prime}\right)$ thus has to be smooth and increasing.

A polynomial least square approximation of the set of key points $\mathcal{K}$ can be used to decrease the noise. We start the approximation with a polynomial of degree two and compute the Euclidean distance 
from $\mathcal{K}$ to the polynomial (least square method), followed by a polynomial of degree three, again computing the Euclidian distance. The difference between these two distances is a measurement of the improvement of the fit, i.e. the larger the difference the greater the improvement. We repeat this evaluation for polynomials of degree three and four, etc. The polynomial approximation, $R(x)$, which leads to the greatest improvement (least square distance) will be used to improve the estimation of $\beta$.

Adjusting $\beta$ value: The acquisition noise leads to a relatively smaller error in the key point $\left(t_{i}, t_{i}^{\prime}\right)$ if $t_{i}$ belongs to a neighborhood of the peak ejection or of the TAC filling rates because the absolute value of the slope is maximal (Fig. 3). Thus, the key points in this neighborhood can be used to compute a new value for $\beta$ that would locate the key point on the previously computed polynomial approximation. For each time sample $t_{i}$ in the neighborhood of the peak ejection or filling rate, the adjusted values for $\beta$ are computed as follows:

$$
\beta=\frac{\log \left(R\left(t_{i}\right)\right)}{\log \left(P\left(t_{i}\right)\right)}
$$

This set of values for $\beta$ is then averaged and the mean is used to adjust the ejection fraction EF roughly approximated before.

\subsubsection{Computing $Q(t)$ polynomial}

the key points give an idea of the smoothness of the acquired data with respect to our model. If the experimental data were noise free, we would expect $Q(t)$ to interpolate all data:

$$
\left\{\begin{array}{l}
Q\left(t_{1}\right)=t_{1}^{\prime}=0 \\
Q\left(t_{2}\right)=t_{2}^{\prime} \\
Q\left(t_{3}\right)=t_{3}^{\prime} \\
Q\left(t_{T-1}\right)=t_{T-1}^{\prime} \\
Q\left(t_{T}\right)=t_{T}^{\prime} \\
Q(1)=1
\end{array}\right.
$$

If some noise is added, the best fit should look like a linear regression of key points. The constraints defined in (3) do not often allow interpolation of all acquired points. So the last step is to compute $Q(x)$ for all real values between 0 and 1, satisfying (3) and corresponding to a good approximation of (5). Hence, let us define $Q(x)$ :

$$
\mathrm{Q}(x)=a_{q} x^{q}+\sum_{i=1}^{n} a_{i} x^{i}, n<q \text { and } n \leq T
$$

The first monomial, $a_{q} \cdot x^{q}$ is added to a polynomial function of degree $n$ to make the condition $Q(1)=1$ possible. 
If we search $Q(x)$ as the least square approximation of (5) on the vector space generated by $\left(x^{q}, x^{n}, x^{n-1}, \ldots, x^{2}, x\right)$, then the set of parameters $\left(a_{q}, a_{n}, \ldots a_{1}\right)$ can be computed by solving the system:

$$
\left(\begin{array}{ccccc}
\sum_{i=1}^{T+1} t_{i}^{2 q} & \sum_{i=1}^{T+1} t_{i}^{q+n} & \ldots & \ldots & \sum_{i=1}^{T+1} t_{i}^{q+1} \\
\sum_{i=1}^{T+1} t_{i}^{q+n} & \sum_{i=1}^{T+1} t_{i}^{2 n} & \sum_{i=1}^{T+1} t_{i}^{2 n-1} & \ldots & \sum_{i=1}^{T+1} t_{i}^{n+1} \\
\sum_{i=1}^{T+1} t_{i}^{q+n-1} & \sum_{i=1}^{T+1} t_{i}^{2 n-1} & \sum_{i=1}^{T+1} t_{i}^{2 n-2} & \ldots & \vdots \\
\vdots & \vdots & \vdots & \ddots & \vdots \\
\sum_{i=1}^{T+1} t_{i}^{q+1} & \sum_{i=1}^{T+1} t_{i}^{n+1} & \ldots & \ldots & \sum_{i=1}^{T+1} t_{i}^{2}
\end{array}\right)\left(\begin{array}{c}
a_{q} \\
a_{n} \\
a_{n-1} \\
\vdots \\
a_{1}
\end{array}\right)=\left(\begin{array}{c}
\sum_{i=1}^{T+1} t_{i}^{\prime} \cdot t_{i}^{q} \\
\sum_{i=1}^{T+1} t_{i}^{\prime} \cdot t_{i}^{n} \\
\sum_{i=1}^{T+1} t_{i}^{\prime} \cdot t_{i}^{n-1} \\
\vdots \\
\sum_{i=1}^{T+1} t_{i}^{\prime} \cdot t_{i}
\end{array}\right)
$$

To satisfy the additional condition $Q(1)=a_{q}+\sum_{i=1}^{n} a_{i}=1$ the set of parameters defining $Q(x)$ are computed by solving the modified matrix equation instead of (7):

$$
\left(\begin{array}{ccccc}
1 & 1 & \ldots & \ldots & 1 \\
\sum_{i=1}^{T+1} t_{i}^{q+n} & \sum_{i=1}^{T+1} t_{i}^{2 n} & \sum_{i=1}^{T+1} t_{i}^{2 n-1} & \ldots & \sum_{i=1}^{T+1} t_{i}^{n+1} \\
\sum_{i=1}^{T+1} t_{i}^{q+n-1} & \sum_{i=1}^{T+1} t_{i}^{2 n-1} & \sum_{i=1}^{T+1} t_{i}^{2 n-2} & \ldots & \vdots \\
\vdots & \vdots & \vdots & \ddots & \vdots \\
\sum_{i=1}^{T+1} t_{i}^{q+1} & \sum_{i=1}^{T+1} t_{i}^{n+1} & \ldots & \ldots & \sum_{i=1}^{T+1} t_{i}^{2}
\end{array}\right)\left(\begin{array}{c}
a_{q} \\
a_{n} \\
a_{n-1} \\
\vdots \\
a_{1}
\end{array}\right)=\left(\begin{array}{c}
1 \\
\sum_{i=1}^{T+1} t_{i}^{\prime} \cdot t_{i}^{n} \\
\sum_{i=1}^{T+1} t_{i}^{\prime} \cdot t_{i}^{n-1} \\
\vdots \\
\sum_{i=1}^{T+1} t_{i}^{\prime} \cdot t_{i}
\end{array}\right)
$$

From a practical standpoint, the method described in this paper was evaluated with $q=10$. For each experimental TAC analyzed, $n$ is set at the highest value smaller than T, for which $Q(t)$ is increasing on $[0,1]$, and which provides the best least square distance between $D\left(t_{i}\right)$ and $E\left(t_{i}\right) i=1,2, \ldots T$.

Figure 4 illustrates the differences between harmonic based methods and the model deformation fit.

\section{ACCURACY EVALUATION}

Six commonly encountered regional time-activity 8-point curves were created and used as original templates for noise superposition (Fig. 5). To take into account the errors due to time sampling, the minimum value of each curve was not included in any sample tested. These curves were designed to produce two different ejection fractions $(65 \%$ and $20 \%)$ and three different end systole times (200 msec, $400 \mathrm{msec}$ and $600 \mathrm{msec}$, with a cardiac frequency normalized at $1 \mathrm{beat} / \mathrm{sec}$ ). The four reference parameters measured for each curve are given in Table 1 Different activity values at end-diastole, $\mathrm{N}$, 
(ranging from 500 to 1500 counts) were used to simulate various statistics. This range covers the usual count rates measured in routine settings. Previous studies and measurements of local statistical noise levels in a reconstructed section using a filtered back projection algorithm $[34,35,36]$ have shown that the order of magnitude of the coefficient of variation of noise linearly depends on the square root of the number of counts. The noise in each TACs was thus assumed to be a Poisson distribution [32].

For each count level $\mathrm{N}$, the rejection method was used to generate 500 different random Poisson deviates of the values of the TAC $\phi_{N}^{k}\left(t_{i}\right), \mathrm{k}=1$ to $500, \mathrm{i}=1$ to 8 [37]. The noise level in each TAC was characterized by the end diastolic coefficients of variation $\left(\mathrm{CV}_{E D}\right)$ of the simulated Poisson deviates (i.e. the inverse of the square root of the count rate at end-diastole). The noise levels simulated in this study correspond to $\mathrm{CV}_{E D}$ ranging from $2.6 \%$ to $4.5 \%$.

To comparatively evaluate the fit described previously, a Fourier transform was performed on simulated noisy TACs. New curves were reconstructed in the time domain using the first, the first two and first three harmonics (resp. methods H1, H2, H3), i.e. the components of frequency $f=\frac{1}{T}, 2 f$ and $3 f$. This is equivalent to filtering by a square-shaped, low-pass filter. The ejection fraction, time of end systole, peak ejection rate, peak filling rate and their time of occurrence were evaluated from the analytic formulae of these filtered curves. These results were compared to the evaluation of the same parameters derived from $D(t)$ (method $\mathrm{M}$ ) with the same previously described reference TAC.

We computed errors made by each method when measuring ventricular parameters from the noisy curves. For each of the original test curves, 500 Poisson deviates were generated for 72 count levels at end-diastole ranging from 500 to 1500 . For the assessment of each ventricular parameter (EF, TES, PFR, PER), we computed the mean and standard deviation of the error obtained using first or multiharmonic Fourier fits and the method described in this paper. These results are given in Table 2 for $20 \%$ EF curves, and Table 3 for $65 \%$ EF curves. The chosen units allow a comparison of errors even if they do not refer to the same original template.

Lastly, as the previously described test curves are based on time samples, they cannot be directly used to evaluate changes in accuracy evaluation of systolic or diastolic parameters when the gated time intervals increase or decrease. Then these parameters were evaluated using the template-based fitting method with 16 time sample test curves. These curves were collapsed to simulate 8-frame data and changes in the evaluation of TES, FES, PER and PFR were measured using the template-based fitting method described in this paper.

\section{Results}

Tables 2 and 3 show mean errors and standard deviations obtained when assessing TES, EF, PER and PFR from noisy TACs using the four tested methods. After checking that all means had a Gaussian distribution, the two methods leading to the two smallest errors were selected and a paired Student test was performed to compare the best two errors. The significance threshold was set at $p=0.005$. When two means are not significantly different they are indicated by “*” in Tables 2 or 3 . In Tables 2 and 3, a bold value denotes a mean error which is not significantly different from zero. 
Lastly, using 16 intervals instead of 8 , the template based fitting method described in this paper leads to average variations, using test curves 1,2,3 (corresponding to curves with 20\% EF), of $3 \mathrm{~ms}$ for TES, $0.3 \%$ for FES, 0.06 EDA.s ${ }^{-1}$ for PER and 0.04 EDA. $\mathrm{s}^{-1}$ for PFR. Average variations with test curves 4,5,6 (corresponding to curves with $65 \% \mathrm{EF}$ ) are $6.1 \mathrm{~ms}$ for TES, 2.9\% for FES, 0.3 EDA.s ${ }^{-1}$ for PER and 0.19 EDA.s ${ }^{-1}$ for PFR.

\section{Discussion \& Conclusion}

In this paper our goal was only to extract reliable parameters from a TAC acquired with a given number of noisy samples. However, the template-based fitting method described in this paper requires relatively accurate endocardial points as input. Consequenctly, the choice of segmentation method used to derive surface points probably affects the amount of noise in the experimental TACs acquired. Software packages have already been proposed for TRVG segmentation [18, 19, 20, 21, 22, 23, 24, 25, 26, 27, 28], using various methods such as manual delineation, thresholding or watersheds. Further clinical validation studies are necessary to evaluate the accuracy of the template-based fitting method described in this paper when input TACs present additional artefacts due to the segmentation algorithm used.

The number of time samples, T, used to acquire TACs has been widely discussed for tomographic gated blood-pool ventriculography (TRVG) [32] or gated SPECT [38, 39, 40]. To ensure reliable measurement with respect to noise, a minimum number of counts must be acquired for each time sample. So $\mathrm{T}$ is directly connected to the exam duration which in routine clinical settings should not exceed 30 to $45 \mathrm{~min}$. In practice, this limitation makes it hard to use $\mathrm{T}$ values greater than 16 .

Our results show a moderate but significant alteration in FES evaluation (compared with the reproducibility of multi-gated angiography methods) with the template-based fitting model when T decreases from 16 to 8 time intervals. This is consistent with previously published results $[38,39,40]$. This alteration can or cannot be accepted depending on the accuracy required by clinical studies. In particular, the method described in this paper is more robust for TES evaluation, so most clinical studies dedicated to assessment of this parameter may be performed with only 8 time intervals, which makes it acceptable in routine settings. On the other hand, accurate evaluation of EF or assessment of diastolic function may require more time intervals. Further clinical studies are necessary to specify each acquisition protocol, depending on the clinical problem involved.

Noise can be reduced by the use of low pass filters, which is what $\mathrm{H} 1, \mathrm{H} 2$, and $\mathrm{H} 3 \mathrm{do}$. Using only the lowest frequency, like $\mathrm{H} 1$, may miss crucial frequencies in retrieving ventricular parameters. The results obtained with the $200 \mathrm{~ms}$ curves are a relevant illustration of this point. Frequencies necessary to create TACs with early TES are higher than the first one, and so measured TES seem to occur much later with H1. PER evaluation is therefore widely underestimated (absolute value). Moreover, large EF errors occur for the same reason. Errors produced with previous parameters lead to an underestimate of PFR values. Using only one frequency does not allow $\mathrm{H} 1$ to reconstruct a wide variety of curves, which is why the standard deviations measured are the lowest. Regarding the large errors in the mean errors evaluated with $\mathrm{H} 1$, the low standard deviations are less a proof of accuracy than the consequence of a 
poor highly constrained fit. Hence, all standard deviations measured for peak rates are equal for a given EF.

Using the first two harmonics (H2) we obtain better results for mean values and standard deviations, but some cases can still lead to major errors. The previous problem is not fully overcome by adding another frequency. The high value of some means indicates that for $\mathrm{H} 2$ the standard deviation error may again be more representative of $\mathrm{H} 2$ constraints than the accuracy of the reconstruction method. If we expect our modeling to be efficient in all cases (even locally), it must be able to fit a large variety of curves.

Adding a third frequency (H3) increases standard deviations but avoids errors, especially for TES and EF measurements. However, the accuracy of PER and PFR measurement decreases relative to H2. The third frequency appears to be useful for reconstructing some shapes, thus avoiding large error because of the constraints observed before.

Another approach to reduce the noise involves the use of non-linear filters (median, morphologic, etc). However, these filters use local information and so cannot be efficient enough when the $\mathrm{T}$ value is low. With the method described in this paper (M), we developed a different approach to fit the acquired curves. First, as we are seeking to generate ventricular ejection curves, we can include $a$ priori knowledge. Indeed, we already know the main characteristics of the curves we are looking for. The method $\mathrm{M}$ uses this a priori knowledge, defined in (1) as a guideline, to fit the acquired data by reshaping a model, thus allowing generation of TACs with more significantly high frequencies. The reference TAC used here was designed to fit experimental curves having one filling and one ejection phase. Deformation of the reference TAC allows satisfactory fits regardless of the phase shift or the magnitude of the experimental TAC. The reference TAC is thus suitable for the analysis of most usual TACs (including severely hypokynetic TACs), except TACs with more than one filling or emptying phase. From a physiologic standpoint, this situation is unrealistic but such curves may be recorded for instance when very noisy TACs are acquired with a severely dyskinetic heart. Increasing the number of shapes that can be generated by a method is a first step to improve the accuracy of a method. The second step is to provide an efficient way to find the best model and to avoid large errors. The means obtained with $\mathrm{M}$ never lead to a large error for TES/EF or for peak rates. Moreover, irrespective of the shape of the noisy experimental TAC, these mean errors do not significantly differ from zero, contrary to $\mathrm{H} 1, \mathrm{H} 2$ or $\mathrm{H} 3$. The standard deviation for TES and EF are mostly equivalent to those obtained with H3. Peak rate standard deviations are higher with the model (because $\mathrm{M}$ does not have any frequency constraint), but mean results for PFR and PER are much better with M than H3.

In conclusion, the method proposed in this paper allows a better fit of noisy TACs acquired in tomographic radionuclide ventriculography than usual multi-harmonic reconstructions. Further clinical studies are now necessary to evaluate its interest in the assessment of wall motion abnormalities among patients. These studies will have to validate methods for 3D noisy TACs generation (such as the method proposed by Vilain et al [29]) and the accuracy of the 3D segmentation of right and left ventricles for each time sample. 


\section{List of table captions}

$1 \quad$ True test curve values for TES,FES,PER and PFR ......................... 17

2 Mean errors and standard deviation of the mean errors obtained for TES, EF, PER and PFR assessment with mono-harmonic $(\mathrm{H} 1)$, multi-harmonics $(\mathrm{H} 2, \mathrm{H} 3)$ or the fit described in this paper $(\mathrm{M})$ for noisy test curves presenting a theoretical EF of 20\%. TES errors are expressed in ms (with heart cycle normalized to one beat/sec), EF errors are expressed as a percentage and peak error units are end

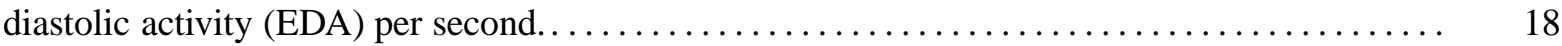

3 Mean errors and standard deviation of the mean errors obtained for TES, EF, PER and PFR assessment with mono-harmonic (H1), multi-harmonics $(\mathrm{H} 2, \mathrm{H} 3)$ or the fit described in this paper (M) for noisy test curves presenting a theoretical EF of $65 \%$. TES errors are expressed in ms (with heart cycle normalized to one beat/sec), EF errors are expressed as a percentage and peak error units are end diastolic activity (EDA) per second. 
TABLE 1

\begin{tabular}{|c|c|c|c|}
\hline TES & EF & PER & PFR \\
\hline $200 m s$ & $20 \%$ & -1.50 EDA..$s^{-1}$ & 0.89 EDA. $s^{-1}$ \\
\hline $400 m s$ & $20 \%$ & -0.75 EDA. $s^{-1}$ & 0.58 EDA. $s^{-1}$ \\
\hline $600 m s$ & $20 \%$ & -0.71 EDA. $s^{-1}$ & 0.75 EDA. $s^{-1}$ \\
\hline $200 m s$ & $65 \%$ & -5.26 EDA. $s^{-1}$ & 2.32 EDA. $s^{-1}$ \\
\hline $400 m s$ & $65 \%$ & -2.42 EDA..$s^{-1}$ & 1.74 EDA..$s^{-1}$ \\
\hline $600 m s$ & $65 \%$ & -2.10 EDA.$s^{-1}$ & 3.39 EDA..$s^{-1}$ \\
\hline
\end{tabular}


TABLE 2

\begin{tabular}{|c|c|c|c|c|}
\hline $200 m s, 20 \%$ & $\mathrm{H} 1$ & $\mathrm{H} 2$ & $\mathrm{H} 3$ & $\mathrm{M}$ \\
\hline TES (ms) & $48.7 \pm 40.9$ & $\mathbf{2 1 . 1} \pm 25.6$ & $6.9 \pm 27.3$ & $\mathbf{1 5 . 9} \pm 44.2$ \\
\hline $\mathrm{EF}(\%)$ & $-6.6 \pm 2.9$ & $-\mathbf{1 . 4} \pm 3.2$ & $\mathbf{1 . 1 4} \pm 3.5$ & $\mathbf{0 . 1 6} \pm 3.7$ \\
\hline PER (EDA. s $^{-1}$ ) & $1.08 \pm 0.09$ & $0.57 \pm 0.2$ & $\mathbf{0 . 1 6} \pm 0.32$ & $\mathbf{0 . 3 7} \pm 0.43$ \\
\hline PFR (EDA. $\left.s^{-1}\right)$ & $-0.47 \pm 0.09$ & $\mathbf{- 0 . 0 7} \pm 0.19$ & $\mathbf{0 . 3 0} \pm 0.3$ & $\mathbf{- 0 . 0 6} \pm 0.33$ \\
\hline "400ms, $20 \%$ & $\overline{\mathrm{H} 1}$ & $\overline{\mathrm{H} 2}$ & $\overline{\mathrm{H} 33}$ & $\overline{\bar{M}}$ \\
\hline TES (ms) & $32.4 \pm 28.2$ & $\mathbf{- 1 . 9 ^ { * }} \pm 37$ & $\mathbf{7 . 8} \pm 57.2$ & $-2.3^{*} \pm 48.1$ \\
\hline $\mathrm{EF}(\%)$ & $-1.66 \pm 2.2$ & $\mathbf{0 . 4 8} \pm 3.0$ & $\mathbf{1 . 8 2} \pm 3.2$ & $\mathbf{0 . 9 0} \pm 3.2$ \\
\hline PER (EDA.s ${ }^{-1}$ ) & $\overline{\mathbf{0 . 1 7}} \pm 0.09$ & $\mathbf{- 0 . 1 2} \pm 0.19$ & $\mathbf{- 0 . 2 8} \pm 0.26$ & $\mathbf{- 0 . 2 0} \pm 0.31$ \\
\hline PFR (EDA. $s^{-1}$ ) & $\mathbf{- 0 . 0 1} \pm 0.09$ & $\mathbf{0 . 0 9} \pm 0.16$ & $\mathbf{0 . 3 3} \pm 0.25$ & $\mathbf{0 . 1 4} \pm 0.25$ \\
\hline 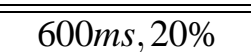 & $\overline{\mathrm{H} 1}$ & $\overline{\mathrm{H} 2}$ & $\overline{\mathrm{H} 3}$ & $\overline{\mathrm{M}}$ \\
\hline TES (ms) & $-\mathbf{- 7 . 0} \pm 28.6$ & $\mathbf{7 . 0} \pm 37.6$ & $-6.2 \pm 42.9$ & $\overline{\mathbf{0 . 1}} \pm 42.4$ \\
\hline $\mathrm{EF}(\%)$ & $-\mathbf{1 . 9 4} \pm 2.8$ & $\mathbf{- 0 . 2 7} \pm 2.9$ & $\mathbf{2 . 0 0} \pm 3.2$ & $0.87 \pm 3.2$ \\
\hline PER (EDA.s ${ }^{-1}$ ) & $\mathbf{0 . 1 5} \pm 0.09$ & $\mathbf{0 . 0 1} \pm 0.18$ & $\mathbf{0 . 2 7} \pm 0.27$ & $\mathbf{- 0 . 0 7} \pm 0.25$ \\
\hline PFR (EDA. $\left.s^{-1}\right)$ & $-0.19 \pm 0.09$ & $\mathbf{0 . 0 3} \pm 0.19$ & $\mathbf{0 . 2 1} \pm 0.24$ & $\mathbf{0 . 1 4} \pm 0.3$ \\
\hline
\end{tabular}


TABLE 3

\begin{tabular}{|c|c|c|c|c|}
\hline $200 m s, 65 \%$ & $\mathrm{H} 1$ & $\mathrm{H} 2$ & $\mathrm{H} 3$ & $\bar{M}$ \\
\hline TES (ms) & $81.4 \pm 9.9$ & $44.2 \pm 6.2$ & $24.2 \pm 6.8$ & $\mathbf{1 5 . 0} \pm 12$ \\
\hline $\mathrm{EF}(\%)$ & $-16.5 \pm 2$ & $-2.5 \pm 2.2$ & $\mathbf{0 . 9} \pm 2.3$ & $\mathbf{0 . 4} \pm 3.4$ \\
\hline PER (EDA.s ${ }^{-1}$ ) & $3.74 \pm 0.06$ & $2.15 \pm 0.14$ & $1.21 \pm 0.27$ & $\mathbf{0 . 4 8} \pm 0.63$ \\
\hline PFR (EDA. $\left.s^{-1}\right)$ & $-0.80 \pm 0.06$ & $\mathbf{0 . 2 0}^{*} \pm 0.19$ & $\mathbf{0 . 2 3} \pm 0.3$ & $\mathbf{0 . 2 0}^{*} \pm 0.42$ \\
\hline $400 m s, 65 \%$ & H1 & $\mathrm{H} 2$ & $\mathrm{H} 3$ & $\bar{M}$ \\
\hline TES (ms) & $29.5 \pm 7.7$ & $\mathbf{- 8 . 6} \pm 8.9$ & $\mathbf{0 . 8} \pm 16.6$ & $\mathbf{- 6 . 5} \pm 15.7$ \\
\hline $\mathrm{EF}(\%)$ & $-5.4 \pm 1.8$ & $\mathbf{- 0 . 6} \pm 2.0$ & $-0.7 \pm 2.1$ & $\mathbf{0 . 5} \pm 2.1$ \\
\hline 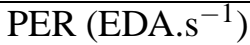 & $0.54 \pm 0.06$ & $\mathbf{- 0 . 2 8} \pm 0.16$ & $\mathbf{- \mathbf { 0 . 2 5 }} \pm 0.29$ & $\mathbf{- \mathbf { 0 . 1 9 }} \pm 0.37$ \\
\hline $\left.\operatorname{PFR}\left(\text { EDA }^{-1}\right)^{-1}\right)$ & $0.13 \pm 0.06$ & $\mathbf{0 . 0 5} \pm 0.19$ & $\mathbf{0 . 2 5} \pm 0.27$ & $\mathbf{0 . 2 0} \pm 0.39$ \\
\hline $600 m s, 65 \%$ & H1 & $\overline{\mathrm{H} 2}$ & $\overline{\mathrm{H} 3}$ & $\bar{M}$ \\
\hline TES (ms) & $-27.7 \pm 8.2$ & $-19.8 \pm 7.5$ & $\mathbf{- 1 3 . 4} \pm 8.4$ & $-7.3 \pm 11.4$ \\
\hline $\mathrm{EF}(\%)$ & $-9.5 \pm 1.8$ & $\mathbf{- 3 . 5} \pm 2.0$ & $\mathbf{- 0 . 2 4} \pm 2.3$ & $\mathbf{- 0 . 0 5} \pm 2.4$ \\
\hline PER (EDA. s $^{-1}$ ) & $0.36 \pm 0.06$ & $\overline{\mathbf{0 . 3 8}} \pm 0.2$ & $\mathbf{- 0 . 4 7} \pm 0.3$ & $\mathbf{0 . 3} \pm 0.3$ \\
\hline PFR (EDA. $\left.s^{-1}\right)$ & $-1.65 \pm 0.06$ & $-0.73 \pm 0.18$ & $\mathbf{0 . 3} \pm 0.3$ & $\mathbf{- 0 . 1 7} \pm 0.39$ \\
\hline
\end{tabular}




\section{List of figure captions}

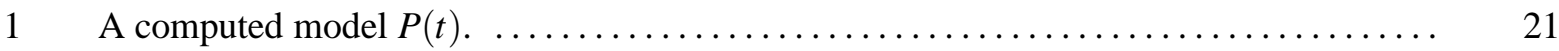

2 Computing key points. The solid curve represents the function $P(t)^{\beta}$ and the dots are the acquired time samples. In this case $\left(t_{i}, u_{i}\right)$ is selected as key point. ................... 22

3 Improving the $\beta$ value: The accuracy of key point evaluation from noisy data is better in the

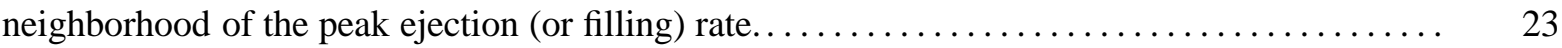

4 A sampled curve (a) reconstructed, after adding noise, with the first harmonic (b), the first two harmonics (c), the first three harmonics (d) and the model deformation (e) ............. 24

5 Test samples for various ejection fractions (EF) and times of end systole (TES). An interpolation between samples is given for visual purposes............................. 25 
FIGURE 1

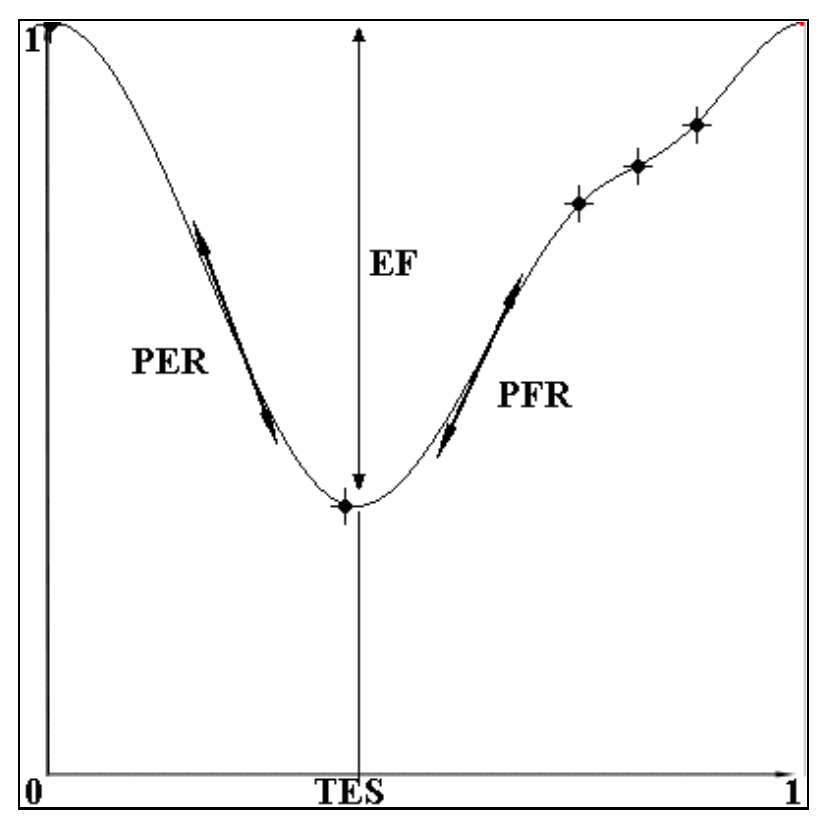


FIGURE 2

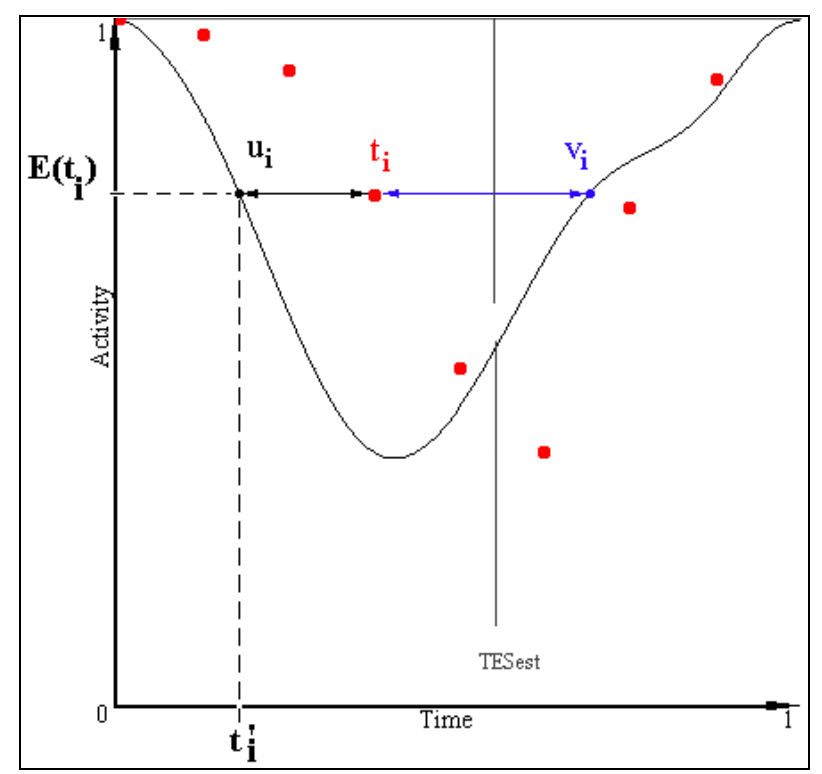


FIGURE 3

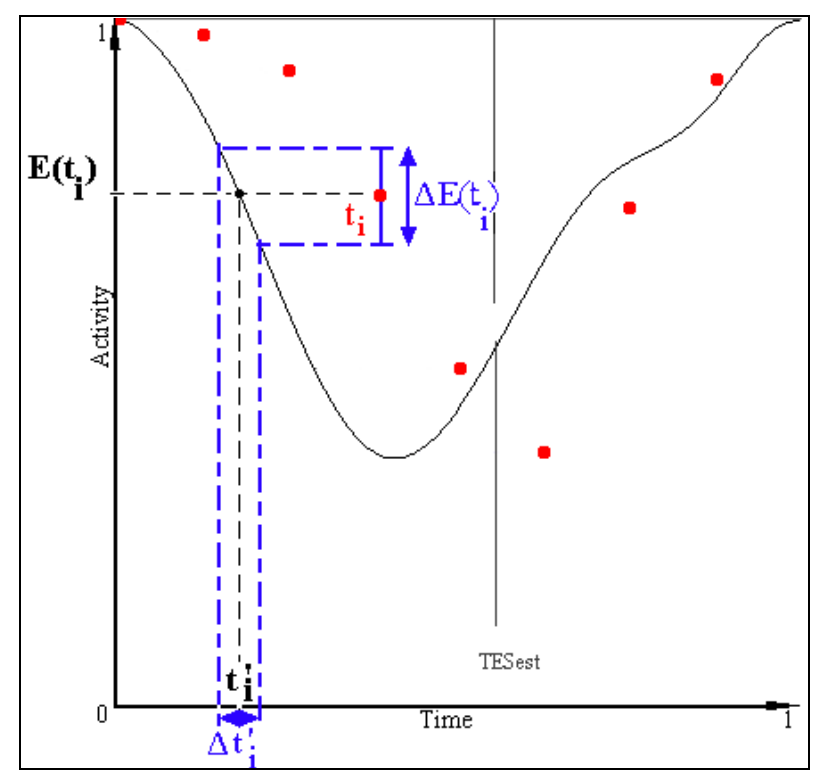


FIGURE 4
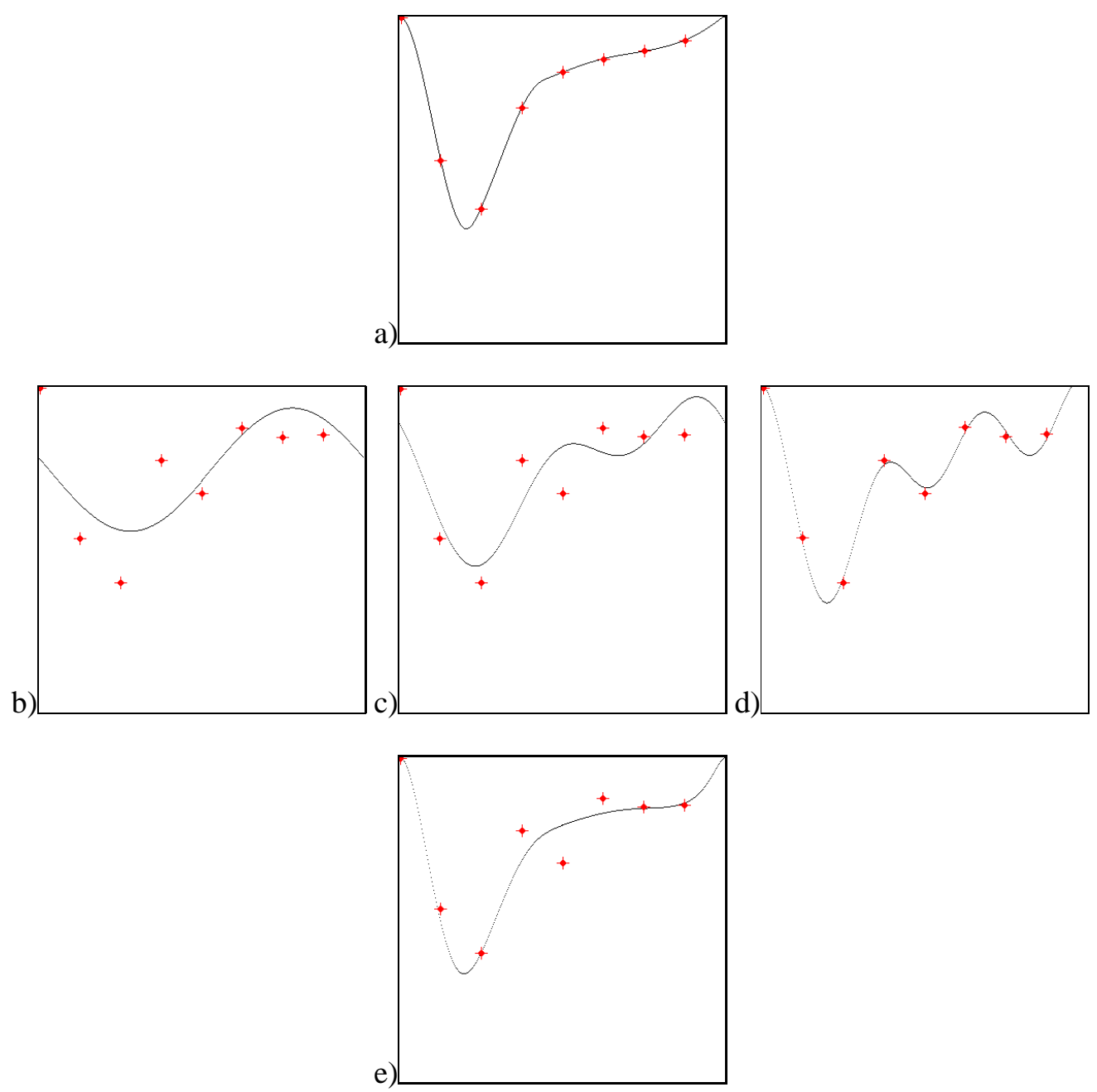
FIGURE 5

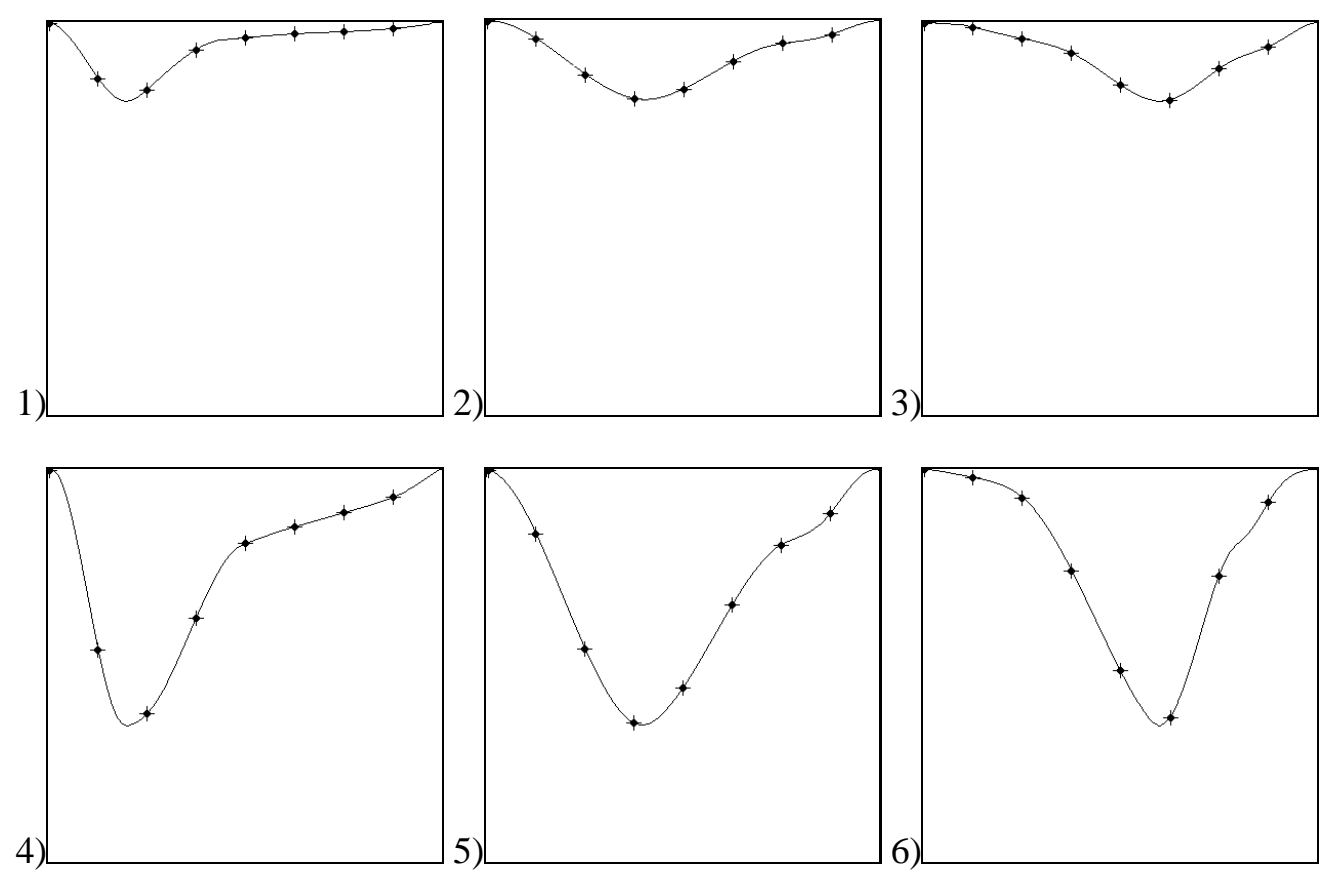




\section{References}

[1] M. Groch, D. Schippers, R. Marshall, P. Groch, and W. Erwin, "Quantitative gated blood pool spect:analysis of 3-dimensional models for the assessment of regional myocardial wall motion." $J$ Nucl Cardiol, vol. 9, pp. 271-284, 2002.

[2] M. Moore, P. Murphy, and J. Burdine, "Ecg-gated emission computed tomography of the cardiac blood-pool," Radiology, vol. 134, pp. 233-235, 1980.

[3] J. Barat, A. Brendel, J. Colle, et al., "Quantitative analysis of left ventricular function using single photon emission tomography," J Nucl Med, vol. 25, pp. 1167-1174, 1984.

[4] K. Nichols, M. Adatepe, and G. Isaacs, "A new scintigraphic method for determining left ventricular volumes," Circulation, vol. 70, pp. 672-680, 1984.

[5] S. Underwood, S. Walton, P. Laming, et al., "Left ventricular volume and ejection fraction determined by gated blood pool emission tomography," Br Heart J, vol. 53, pp. 216-222, 1985.

[6] J. Corbett, D. Jansen, S. Lewis, et al., "Tomographic gated blood pool radionuclide ventriculography : analysis of wall motion and left ventricular volumes in patients with coronary artery disease," J Am Coll Cardiol, vol. 6, pp. 349-358, 1985.

[7] S. Underwood, S. W. S, P. Ell, et al., "Gated blood-pool emission tomography : new technique for the investigation of cardiac structure and function," Eur J Nucl Med, vol. 10, pp. 332-337, 1985.

[8] M. Stadius, D. Williams, G. Harp, et al., "Left ventricular volume determination using single photon emission computed tomography," Am J Cardiol, vol. 55, pp. 1185-1191, 1985.

[9] S. Bunker, M. Hartshorne, W. Schmidt, et al., "Left ventricular volume determination by single photon emission computed tomography," Am J Roentgenol, vol. 144, pp. 295-298, 1985.

[10] G. C. GR, M. Graham, D. Kirk, et al., "Measurement of left ventricular volume using singlephoton emission computed tomography," Am J Cardiol, vol. 56, pp. 781-786, 1985.

[11] J. Gill, R. Moore, N. Tamaki, et al., "Multigated blood-pool tomography : new method for the assessment of left ventricular function," J Nucl Med, vol. 27, pp. 1916-1924, 1986.

[12] B. Chin, D. Bloomgarden, W. Xia, et al., "Right and left ventricular volume and ejction fraction by tomographic gated blood-pool scintigraphy," J Nucl Med, vol. 38, pp. 942-948, 1997.

[13] P. Pretorius, W. Xia, M. King, B. Tsui, T. Pan, and B. Villegas, "Evaluation of right and left ventricular volume and ejection fraction using a mathematical cardiac torso phantom," $J \mathrm{Nucl}$ Med, vol. 38, pp. 1528-1535, 1997. 
[14] M. Cerqueira, G. Harp, and J. Ritchie, "Quantitative gated blood pool tomographic assessment of regional ejection fraction : definition of normal limits," JACC, vol. 20, pp. 934-941, 1992.

[15] T. Ohtake, J. Nishikawa, K. Machida, et al., "Evaluation of regurgitant fraction of the left ventricle by gated cardiac blood-pool scanning using spect," J Nucl Med, vol. 28, pp. 19-24, 1987.

[16] K. Nakajima, H. Bunko, A. Tada, et al., "Nuclear tomographic phase analysis localization of accessory conduction pathway in patients with wolff-parkinson-white syndrome," Am Heart J, vol. 109, pp. 809-815, 1985.

[17] R. Itti, D. Casset, L. Philippe, J. Fauchier, P. Cosnay, and R. Huguet, "Characterization of right or left ventricular contraction heterogeneity using fourier phase analysis," Eur J Nucl Med, vol. 14, pp. 196-202, 1988.

[18] S. Kriekinge, D. Berman, and G. Germano, "Automatic quantification of left ventricular ejection fraction from gated blood-pool spect," J Nucl Cardiol, vol. 6, pp. 498-506, 1999.

[19] G. Germano and D. Berman, “The right stuff,” J Nucl Cardiol, vol. 9, pp. 226-228, 2002.

[20] D. Mariano-Goulart, H. Collet, P. Kotzki, M. Zanca, and M. Rossi, "Semi-automatic segmentation of gated blood pool emission tomographic images by watershed : application to the determination of right and left ejection fractions," Eur J Nucl Med, vol. 25, pp. 1300-1307, 1998.

[21] D. Mariano-Goulart, H. Collet, M. Eberle, et al., "Routine measurements of right and left ejection fractions thanks to the segmentation of gated blood pool tomographic images by a watershed algorithm," Eur J Nucl Med, vol. 26, p. 1078, 1999.

[22] D. Mariano-Goulart, C. Piot, V. Boudousq, F. Raczka, F. Comte, M. Eberlé, M. Zanca, P. Kotzki, J. Davy, and M. Rossi, "Routine measurements of left and right ventricular output by gated blood pool emission tomography in comparison with thermodilution measurements: a preliminary study," Eur J Nucl Med, vol. 28, pp. 506-513, 2001.

[23] C. Vanhove, N. Walgraeve, F. D. Geeter, and P. Franken, "Gated myocardial perfusion tomography versus gated blood pool tomography for the calculation of left ventricular volumes and ejection fraction," Eur J Nucl Med Mol Imaging, vol. 29, pp. 735-741, 2002.

[24] C. Vanhove and P. Franken, "Left ventricular ejection fraction and volumes from gated blood pool tomography: comparison between two automatic algorithms that work in three-dimensional space," J Nucl Cardiol, vol. 8, pp. 466-471, 2001.

[25] C. Vanhove, P. Franken, M. Defrise, A. Momen, H. Everaert, and A. Bossuyt, "Automatic determination of left ventricular ejection fraction from gated blood-pool tomography," J Nucl Med, vol. 42, pp. 401-407, 2001. 
[26] K. Nichols, R. Saouaf, A. Ababneh, R. Barst, M. Rosenbaum, M. Groch, et al., "Validation of spect equilibrium radionuclide angiograph right ventricular parameters by cardiac magnetic resonance imaging," J Nucl Cardiol, vol. 9, pp. 5-12, 2002.

[27] E. DePuey, K. Nichols, and C. Dobrinsky, "Left ventricular ejection fraction assessed from gated technetium-99m-sestamibi spect," J Nucl Med, vol. 34, pp. 1871-1876, 1993.

[28] D. Daou, F. Harel, B. Helal, T. Fourne, P. Colin, R. Lebtahi, D. Mariano-Goulart, M. Faraggi, M. Slama, and D. L. Guludec, "Electrocardiographically gated blood-pool spect and left ventricular function: comparative value of 3 methods for ejection fraction and volume estimation," $\mathrm{J} \mathrm{Nucl}$ Med, vol. 42, pp. 1043-1049, 2001.

[29] D. Vilain, D. Daou, D. Casset-Senon, M. Faraggi, and D. L. Guludec, "Optimal 3-dimensional method for right and left ventricular fourier phase analysis in electrocardiography-gated bloodpool spect," J Nucl Cardiol, vol. 8, pp. 371-378, 2001.

[30] S. Bacharach, M. Green, D. Vitale, G. White, M. Douglas, R. Bonow, and S. Larson, "Optimum fourier filtering of cardiac data: a minimum-error method," J Nucl Med, vol. 24, pp. 1176-1184, 1983.

[31] H. Valette, M. Bourguignon, M. Gregoire, P. Merlet, O. Pascal, P. Briandet, D. L. Guludec, and A. Syrota, "Filters and fourier analysis of gated blood pool studies: a search for the optimal combination," Phys Med Biol, vol. 35, pp. 1-9, 1990.

[32] J. Machac, S. Horowitz, D. Broder, and S. Goldsmith, "Accuracy and precision of regional multiharmonic fourier analysis of gated blood pool images," J Nucl Med, vol. 25, pp. 1294-1299, 1984.

[33] H. Valette, M. Bourguignon, P. Merlet, M. Gregoire, P. Briandet, and A. Syrota, "The value of pixel per pixel multiharmonic fourier analysis to assess left ventricular function," Nucl Med Comm, vol. 13, pp. 137-141, 1992.

[34] R. Huesman, "The effects of a finite number of projection angles and finite lateral sampling of projections on the propagation of statistical errors in transverse section reconstrution." Phys. Med. Biol., vol. 22, pp. 511-521, 1977.

[35] T. Budinger, S. Derenzo, W. Greenberg, G. Gullberg, and R. Huesman, "Quantitative potentials of dynamic emission computed tomography," J. Nucl. Med., vol. 19, pp. 305-315, 1978.

[36] G. J. Gillen, "A simple method for the measurement of local statistical noise levels in spect," Phys. Med. Biol, vol. 37, pp. 1573-1579, 1992.

[37] W. Press, S. Teukolsky, W. Vetterling, and B. Flannery, Numerical recipes in C, 1994, pp. $293-$ 295. 
[38] S. kumita, K. Cho, H. Nakajo, M. Toba, M. Uwamori, S. Mizumura, T. Kumazaki, J. Sano, S. Sakai, and K. Munakata, "Aassessment of left ventricular diastolic function with electrocardiography-gated myocardial perfusion spect: comparison with multigated equilibrium radionuclide angiography." J Nucl Cardiol, vol. 8, no. 5, p. 568574, 2001.

[39] M. Kikkawa, T. Nakamura, K. Sakamoto, H. Sugihara, A. Azuma, T. Sawada, C. Okuyama, Y. Ushijima, and T. Nishumura, "Assessment of left ventricular disatolic function from quantitative electrocardiographic-gated 99mtc-tetrofosmin myocardial spet." Eur J Nucl Med, vol. 28, no. 10, pp. 1579-1583, oct 2001.

[40] T. Higuchi, K. Nakajima, J. Taki, S. Kinuya, H. Bunko, and N. Tonami, "Assessment of left ventricular systolic and diastolic function based on the edge detection method with myocardial ecg-gated spet," Eur J Nucl Med, vol. 28, no. 10, pp. 1512-1516, Oct 2001. 